\title{
O papel do enfermeiro dentro do contexto da assistência indígena: uma revisão de
}

\section{literatura}

\author{
The role of nurses within the context of indigenous assistance: a literature review \\ El papel de las enfermeras en el contexto de la asistencia a los indígenas: una revisión de la literatura
}

Recebido: 17/11/2021 | Revisado: 26/11/2021 | Aceito: 03/12/2021 | Publicado: 13/12/2021

\author{
Angelina de Oliveira Lima \\ ORCID: https://orcid.org/0000-0003-4425-8049 \\ Enfermeira, Brasil \\ E-mail: angelinaolima95@gmail.com \\ Amanda Thyanne Sales de Sousa \\ ORCID: https://orcid.org/0000-0001-8940-1035 \\ Centro Universitário FAMETRO, Brasil \\ E-mail: amandatssousa247@gmail.com
}

\begin{abstract}
Resumo
Objetivo: Definir o papel dos profissionais enfermeiros acerca das atribuições dentro do contexto indígena. Métodos: Trata-se de um estudo descritivo exploratório, no qual a metodologia escolhida foi Revisão Integrativa de Literatura, buscados nas bases de dados de bibliotecas virtuais como: Scielo (Scidentific Eletrônica Library Online), Lilacs (Literatura Latino-Americana e do Caribe em Ciências de Saúde), BVS (Biblioteca Virtual de Saúde), Medline (Sistema Online de Análise e Recuperação de Literatura Médica) e Pubmed (Biblioteca Nacional de Medicina dos EUA [NLM]). Resultados: $\mathrm{O}$ enfermeiro exerce um papel primordial na assistência à saúde indígena, mas pode-se observar que para prestar essa assistência, encontram-se certas dificuldades como o acesso. Mesmo diante das falhas de forma defasada na cobertura no contexto de prestação de serviços ao indígena, os profissionais exercem seu papel da melhor forma cabível, mesmo que haja muitas vezes déficit em capacitação por parte dos órgãos e desconhecimento das culturas das etnias onde atuam. Considerações Finais: No desencadeamento do trabalho da enfermagem, encontram-se diversos desafios quanto ao contexto de prestação na assistência indígena. Conclui-se uma importância quanto a melhoria nas implementações das políticas de forma compreensiva pelos gestores das três esferas ao implementar estas políticas no decorrer da assistência a comunidade local.
\end{abstract}

Palavras-chave: Saúde Indígena; Saúde Pública; Atribuições; Enfermagem; Papel do Enfermeiro.

\begin{abstract}
Objective: To define the role of nursing professionals regarding assignments within the indigenous context. Methods: It is an exploratory descriptive study, in which the methodology chosen was Integrative Literature Review, searched in the databases of virtual libraries such as: Scielo (Scidentific Eletrônica Library Online), Lilacs (Latin American and Caribbean Literature in Sciences of Health), VHL (Virtual Health Library), Medline (Medical Literature Analysis and Retrieval System Online) and Pubmed (U.S. National Library of Medicine [NLM]). Results: The nurse plays a primary role in assisting indigenous health, we observe that to provide this assistance, we encounter certain difficulties, such as access, but, even in the face of the failures in coverage in the context of providing services to the indigenous, since they are found in a delay, professionals play their role in the best way possible, even though there is often a lack of training on the part of the agencies and ignorance of the cultures of the ethnic groups where they work. Final Considerations: In the unleashing of nursing work, there are several challenges regarding the context of provision in indigenous care. It concludes an importance regarding the improvement in the implementations of the policies in a comprehensive way by the managers of the three spheres when implementing these policies during the assistance to the local community.
\end{abstract}

Keywords: Indigenous health; Public health; Duties; Nursing; Nurse's role.

\section{Resumen}

Objetivo: Definir el rol de las enfermeras profesionales con respecto a las asignaciones dentro del contexto indígena. Métodos: Se trata de un estudio descriptivo exploratorio, en el que la metodología elegida fue Revisión de Literatura Integrativa, buscada en las bases de datos de bibliotecas virtuales como: Scielo (Biblioteca Scidentific Eletrônica Online), Lilacs (Literatura Latinoamaricana y Caribeña en Ciencias de la Salud), BVS (Biblioteca Virtual en Salud), Medline (Sistema de análisis y recuperación de literatura médica en línea) y Pubmed (Biblioteca Nacional de Medicina de EE. UU. [NLM]). Resultados: La enfermera juega un rol primordial en la atención de la salud indígena, observamos que para brindar esta asistencia, encontramos ciertas dificultades, como el acceso, pero, aún ante fallas en la contexto de la prestación de servicios a los indígenas, ya que se encuentran de manera tardía, los profesionales ejercen su rol de la mejor manera posible, aunque muchas veces existe una falta de capacitación por parte de las 
agencias y el desconocimiento de las culturas de los grupos étnicos donde laboran. Consideraciones Finales: En el desencadenamiento del trabajo de enfermería, existen varios desafíos en cuanto al contexto de la prestación en el cuidado indígena. Se concluye una importancia en cuanto a la mejora en la implementación de las políticas de manera integral por parte de los gestores de los tres ámbitos al implementar estas políticas durante la asistencia a la comunidad local.

Palabras clave: Salud indígena; Salud pública; Deberes; Enfermería; Papel de la enferma.

\section{Introdução}

A Atenção Primária a Saúde (APS), também conhecida por Atenção Básica à Saúde (AB), é o principal meio de entrada para o Sistema de Saúde, ou seja, é a primeira assistência prestada aos usuários que buscam atendimento. A Estratégia Saúde da Família (ESF) é um dos programas multidisciplinares voltado para a saúde, sendo um modelo de assistência implementado pelo Ministério da Saúde (MS), cujo principal objetivo é a prestação a assistência à saúde da família (Giovanella \& Mendonça, 2014).

Em seguimento deste objetivo, a ESF busca a implantação de vínculos com a comunidade, possibilitando um elo entre equipe e sociedade, oportunizando um compromisso contínuo de assistência ao usuário e população, assim como responsabilidade pelos cuidados para com a saúde, além de se caracterizar como uma estratégia inovadora de assistir à saúde (Gelinski Crog, 2011).

A atenção voltada para a assistência à Saúde Indígena cumpre-se no âmbito do Subsistema de Atenção à Saúde Indígena, instituída pela Lei ${ }^{\circ}$ 9.836/1999, estruturado pelo Sistema Único de Saúde (SUS), administrado pela Secretaria Especial de Saúde Indígena (SESAI), órgão este encarregado de gerenciar uma rede de tarefas da APS (Diehl et al., 2012\& Fundação Nacional De Saúde, 2002).

A assistência prestada à Saúde Indígena no nível da AB é exercida por integrantes de Equipes Multidisciplinares de Saúde Indígena, conhecido como EMSI, essa equipe é composta por enfermeiros, médicos, odontólogos, técnicos e auxiliares de enfermagem, agentes indígenas de saúde (AIS), entre outros profissionais (Vieira et al., 2013; Ferreira, 2013; Nan Greenwoo \& Raymond Smith, 2015), e age como um dispositivo para o funcionamento a saúde com foco na vigilância em saúde (Cardoso \& Langdon, 2015), prestando assistência contínua e programada, de acordo com as diretrizes da ESF pelo MS (Garnelo \& Pontes, 2012; Brasil, 2013).

Existem princípios legais que garantem a assistência e o cuidado integral à saúde da população indígena, amparando sua diferença cultural, histórica, política e diversidade social. A Política Nacional da Atenção à Saúde dos Povos Indígenas (PNASPI) aprovada pela Portaria/MS no 254 de 31/2/2002 sugere o reconhecimento de sua medicina e o direito dos povos indígenas à sua cultura (Fundação Nacional de Saúde, 2002).

O sistema voltado para a saúde indígena é um sistema organizado a partir de 34 (trinta e quatro) Distritos Sanitários Especiais Indígenas, conhecido como DSEI, distribuídos em todo Território Nacional, ocupado por unidades gestoras e uma área territorial fixa a partir de critérios geográficos, habitantes e étnico-culturais, responsáveis por desenvolver trabalhos como de promoção, precaução e equilíbrio na saúde e agravos com base nos perfis epidemiológicos e específicos culturais locais (Brasil, 2002).

Os DSEI's recebem diferentes etnias em seus territórios com pessoas aldeadas; os postos de saúde nas aldeias PolosBase e casas de Apoio a saúde do índio, juntamente com a EMSI abrangem toda essa população, ofertando uma atenção íntegra e diferenciada. (Brasil, Portaria $\mathrm{N}^{\circ} 2.656$, de 17 de outubro de 2007).

No decorrer dos anos, houve avanços no modelo de saúde indígena, com suas respectivas implementações nas políticas de saúde no âmbito da saúde indígena e, ainda nos dias atuais, encontram-se problemas como falta de um sistema de informação eficaz, dificuldade de união com a rede de referência do SUS, falta de profissionais capacitados para atuar em um contexto intercultural, alta rotatividade profissional, entre outros impasses (Diehl et al., 2012; Langdon \& Diehl 2007). 
Tais estorvos nos mostram a necessidade de uma contínua avaliação no que diz respeito ao modelo da assistência prestada, assim como na articulação com os sistemas de saúde municipais e isso faz com que haja urgência em implementar instrumentos constituídos que sejam aptos para averiguar o subsistema de saúde indígena e os serviços de saúde pública ofertados nos municípios, de modo que essas pressuposições da assistência diferenciada e a forma da atenção primária se desencadeiam no dia a dia não se conflitem (Rocha, et al., 2020).

$\mathrm{Na}$ atuação da enfermagem no sistema de saúde, observamos que a enfermagem traz consigo grandes desafios e responsabilidades, no entanto, busca constantemente aprimorar seus conhecimentos teóricos-científicos, assim como conhecer as políticas e aspectos étnicos-culturais das diferentes etnias que compõem o Subsistema de Atenção à Saúde Indígena, bem como a assistência prestada na prática, caracterizada pelas diversas ações de saúde, conhecida como Pluralidade Terapêutica (Silva et al., 2003; Vieira, et al., 2013). No processo de cuidar centrado no cliente, é imprescindível que o profissional enfermeiro reconheça a diversidade cultural, o processo saúde-doença e as experiências dos sujeitos assistidos, para que se possa compreender e estabelecer a assistência diferenciada destinada ao usuário indígena (Langdon, 2007).

O processo de trabalho da Enfermagem vem se modificando, trazendo consigo um olhar holístico seja do usuário à família ou comunidade até ações estratégicas de educação em saúde no planejamento do cuidado em saúde. Instituído pela Lei $n^{\circ}$ 7.498/86, (Brasil 1986), a enfermagem tem evoluído no equilíbrio de suas tarefas, previstas no Regulamento do Exercício Profissional, assim como no Código de Ética dos Profissionais de Enfermagem, discorrido na Resolução COFEN (2008).

$\mathrm{Na}$ assistência prestada ao indígena, tornar o trabalho da enfermagem com a prática social representa compreender em seu exercício que não existe apenas uma enfermagem, mas um leque, visto que seus atos se adaptam, se transformam, se reproduzem de acordo com a dinâmica imposta ao cenário encontrado. (Langdon, 2007; Fundação Nacional de Saúde, 2002).

O exercício da enfermagem frente as suas atribuições na atenção à Saúde Indígena tornam-se um trabalho dinâmico e vivo, com atitudes que criam vínculos profissional e paciente, integrando a micropolítica da assistência prestada, sendo capaz de configurar o processo do cuidar ao cliente assistido (Ceccin \& Merly, 2009).

De acordo com a temática, essa pesquisa traz como objetivo geral definir o papel dos profissionais enfermeiros dentro do contexto da assistência na saúde indígena.

A abordagem do enfermeiro na atenção à assistência indígena é uma prática excepcional, onde exige conhecimento técnico e principalmente, ético-cultural exercida com responsabilidade conforme as políticas públicas e atribuições profissionais, uma vez que se constatou distinção neste contexto.

Portanto, as investigações de suas práticas provem da evolução para o exercício integro da profissão dentro do contexto indígena, onde os profissionais serão providos de autonomia, segurança em seguimento deste estudo, assim como capacitados e envolvidos na cultura de cada etnia a ser trabalhada.

Conforme a lei 7.142/90 a atribuição do enfermeiro diz que o exercício profissional garante ao profissional a consulta de enfermagem, a prescrição ou transcrição de medicamentos de acordo com o RENAME municipal, assim como solicitar exames complementares. A AB possui uma amplitude de cuidados e de forma diferenciada de acordo com as políticas de saúde indígena. Descrita na Lei ${ }^{\circ}$ 9.836/1999, a assistência deve ser exercida com habilidade nos campos de gerenciamento, raciocínio clínico, planejamento, comunicação, conhecimento teórico-cientifico em doenças infectocontagiosas, imunização, saúde mental, problemas de pele, hipertensão, diabetes, entre outras morbidades. Ferreira et al., 2018)

\section{Metodologia}

Trata-se de um estudo descritivo exploratório, por meio de revisão integrativa de literatura, no qual este método nos possibilita um abrangente conhecimento direcionado ao tema, ajudando-nos na aplicabilidade de resultados no conhecimento teórico bem como na prestação da assistência (Souza et al., 2010). 
Este trabalho é seguido por algumas etapas, sendo estas: formação de hipótese, busca no meio da literatura, coleta de dados, análise dos artigos, interpretação de resultados e discussão afim de definir sua finalidade descrita anteriormente no objetivo desta pesquisa.

A busca bibliográfica foi realizada no primeiro semestre de 2021, utilizando-se base de dados de bibliotecas virtuais como: SciELO (Scidentific Eletrônica Library Online), LILACS (Literatura Latino-Americana e do Caribe em Ciências da Saúde), BVS (Biblioteca Virtual de Saúde), Medline (Sistema Online de Análise e Recuperação de Literatura Médica) e Pubmed (Biblioteca Nacional de Medicina dos EUA [NLM], por meio dos seguintes descritores: "Saúde Indígena", "Atribuições", "Enfermeiros", "Assistência Indígena" e "Papel do Enfermeiro". Os critérios de elegibilidade utilizado no trabalho foram respectivamente: artigos publicados nos últimos 10 anos, nos idiomas português, inglês e espanhol, completos e gratuitos. Os critérios de ilegibilidade foram: teses, mestrados, artigos em formato de resumo e dissertações.

$\mathrm{Na}$ busca de atingir nosso objetivo, definimos a seguinte questão norteadora: qual o papel do enfermeiro dentro do contexto indígena, diante das políticas públicas?

\section{Resultado e Discussão}

Quanto aos materiais para a realização de análise do estudo foram encontrados no total de 103 artigos, com a utilização dos seguintes descritores "saúde indígena and saúde pública", "atribuições and enfermagem" e "papel do enfermeiro". Ao realizarmos uma filtragem minuciosa com os critérios de elegibilidade e inegibilidade, obtivemos uma amostra final de 22 artigos completos relevantes ao tema. Elaboramos uma planilha, com as seguintes informações: número, título do artigo, autor, ano, idioma, plataforma, resultado alcançado, conforme mostra o Quadro 1.

Quadro 1: Artigos selecionados.

\begin{tabular}{|c|c|c|c|c|c|c|}
\hline $\mathrm{N}^{\circ}$ & Título & Autor & Ano & Idioma & Plataforma & Resultado \\
\hline 1 & $\begin{array}{l}\text { Enfermagem em } \\
\text { Saúde Indígena: } \\
\text { aplicando as } \\
\text { Diretrizes } \\
\text { Curriculares. }\end{array}$ & $\begin{array}{c}\text { Silva NC, } \\
\text { Gonçalves MJF, } \\
\text { Neto DL. }\end{array}$ & 2003 & Português & Scielo & $\begin{array}{l}\text { Para uma atribuição adequada do enfermeiro frente a Saúde Indígena é } \\
\text { essencial o entendimento sobre o processo saúde-doença, incluindo o } \\
\text { aspecto étnico-cultural, visto que o profissional precisa estar capacitado } \\
\text { para atuar na atenção básica à Saúde Indígena, assim como realizar busca } \\
\text { ativa quanto aos problemas encontrados, planejar ações voltadas para } \\
\text { assistência indígena bem como conhecer as plantas medicinais para } \\
\text { contribuir para uma melhor qualidade de atendimento aos índios assim } \\
\text { como criar um vínculo com a população indígena. }\end{array}$ \\
\hline 2 & $\begin{array}{l}\text { Atributos da } \\
\text { Atenção Primária } \\
\text { à Saúde no } \\
\text { contexto da } \\
\text { Saúde Indígena. }\end{array}$ & $\begin{array}{l}\text { Rocha ESC, } \\
\text { Toledo NN, Pina } \\
\text { RMP, Fausto } \\
\text { MCR, D’Viana } \\
\text { AL, Lacerda, } \\
\text { RA. }\end{array}$ & 2020 & Português & Lilacs & $\begin{array}{l}\text { Segundo estudos, observam-se a dificuldade de acesso da população } \\
\text { indígena frente aos serviços de saúde, visto que há variados pontos que } \\
\text { compõe a este impasse voltado para o fluxo de conhecimento da } \\
\text { população, podendo ser citados à alta taxa de rotatividade dos } \\
\text { profissionais na assistência, poucos profissionais capacitados, baixo nível } \\
\text { de conhecimento dos profissionais perante a família do cliente, assim } \\
\text { como pouca comunicação entre o profissional e o usuário. }\end{array}$ \\
\hline 3 & $\begin{array}{l}\text { Promoção da } \\
\text { saúde na } \\
\text { comunidade } \\
\text { Indígena } \\
\text { Pankararu. }\end{array}$ & $\begin{array}{c}\text { Oliveira JWB, } \\
\text { Aquino JM, } \\
\text { Monteiro EMLM }\end{array}$ & 2012 & Português & Scielo & $\begin{array}{l}\text { Alguns estudos apontam que há uma descontinuidade nas ações frente a } \\
\text { assistência indígena, ao acesso básico de saúde, afetando até mesmo a } \\
\text { aproximação da sociedade indígena e o SUS. Nota-se que há pouca } \\
\text { assistência prestada de forma humanizada, vínculo entre equipe de saúde e } \\
\text { comunidade indígena, carência no acesso a informação de saúde sobre as } \\
\text { doenças existentes e que acometem a comunidade, assim como não há } \\
\text { informes de como prevenir, tratar e até mesmo o meio de contágio, } \\
\text { deixando faltar uma realização no que diz respeito a educação em saúde } \\
\text { para a população indígena. }\end{array}$ \\
\hline 4 & $\begin{array}{l}\text { Atitudes, } \\
\text { conhecimentos e } \\
\text { habilidades para } \\
\text { o trabalho do } \\
\text { enfermeiro no } \\
\text { Parque Indígena } \\
\text { do Xingu. }\end{array}$ & $\begin{array}{l}\text { Martins JCL, } \\
\text { Martins CL, } \\
\text { Oliveira LSS. } \\
\text { Ministério da } \\
\text { Saúde (BR); } \\
\text { Silva CD; Silva } \\
\text { CB; Lima VV. } \\
\end{array}$ & 2020 & Inglês & Scielo & $\begin{array}{l}\text { A enfermagem cada vez mais vem se firmando na organização das equipes } \\
\text { da EMSI dos DSEI's. A atuação e competência do enfermeiro é definido a } \\
\text { partir dos diferentes ambientes e campo de atuação visto que o mesmo está } \\
\text { constantemente em contato com os diversos pontos de vista do processo } \\
\text { saúde-doença, portando, deve-se realizar uma melhor assistência de } \\
\text { enfermagem frente a população indígena, o profissional deve-se junto a } \\
\text { equipe multiprofissional, planejar ações de trabalho coletivo e de forma } \\
\text { colaborativa a comunidade, incluindo ações no seu campo de atuação que } \\
\text { fazem com que haja interpretação sobre o desenvolvimento de } \\
\text { adoecimento e tratamento, assim como desenvolver ações educativas que } \\
\text { façam com que os próprios profissionais se preparem no ambiente onde }\end{array}$ \\
\hline
\end{tabular}




\begin{tabular}{|c|c|c|c|c|c|c|}
\hline & & & & & & atuam. \\
\hline 5 & $\begin{array}{l}\text { Singularidades } \\
\text { culturais: o } \\
\text { acesso do idoso } \\
\text { indígena aos } \\
\text { serviços públicos } \\
\quad \text { de saúde. }\end{array}$ & $\begin{array}{c}\text { Borgui AC, } \\
\text { Alvarez AM, } \\
\text { Marcon SS, } \\
\text { Carreira L. } \\
\text { Cardoso AM, } \\
\text { Coimbra Junior } \\
\text { CEA, Tavares } \\
\text { FG; Brasil. } \\
\end{array}$ & 2015 & Inglês & Scielo & $\begin{array}{l}\text { De acordo com as diretrizes e princípios do SUS, o acesso atenção integral } \\
\text { à saúde é garantido, contemplando suas diferentes diversidades, mas, } \\
\text { mesmo assim, ainda se encontram dificuldades de acesso aos serviços de } \\
\text { saúde por meio da população indígena, ocasionando assim um impasse } \\
\text { encontrado pela população frente a assistência indígena. Estudos apontam } \\
\text { que o acesso por meio do indígena ainda é pouco tratado, constatado } \\
\text { quando observamos os pontos de distância entre residência e centro de } \\
\text { saúde, assim como o horário de funcionamento, a demora no atendimento, } \\
\text { entre outros pontos. Portanto, cabe ressaltar que há uma necessidade de } \\
\text { reorganizar o fluxo de atendimento frente a assistência capaz de permitir } \\
\text { melhores recursos ao atendimento destes. }\end{array}$ \\
\hline 6 & $\begin{array}{l}\text { Assistência à } \\
\text { população } \\
\text { indígena: } \\
\text { dificuldades } \\
\text { encontradas por } \\
\text { enfermeiro } \\
\end{array}$ & $\begin{array}{c}\text { Marinelle NP, } \\
\text { Nascimento } \\
\text { DP,Costa AIP, } \\
\text { Posso MBS, } \\
\text { Araújo LP }\end{array}$ & 2012 & Português & BVS & $\begin{array}{l}\text { Observa-se que mesmo com a existência de políticas de saúde as ações } \\
\text { preventivas não estão sendo aplicadas pelos profissionais de saúde } \\
\text { atuantes da área, o que gera insegurança na assistência da enfermagem por } \\
\text { parte dos profissionais. Mesmo diante das dificuldades, a enfermagem está } \\
\text { sempre presente no cuidado à saúde, não medindo esforços para fornecer } \\
\text { cuidados à população indígena, mesmo que muitas vezes haja despreparo e } \\
\text { falta de capacitação para atender uma população tão peculiar. }\end{array}$ \\
\hline 7 & $\begin{array}{l}\text { A atuação do } \\
\text { enfermeiro sobre } \\
\text { práticas de } \\
\text { cuidados } \\
\text { afrodescendentes } \\
\text { e indígenas }\end{array}$ & $\begin{array}{l}\text { Lima MRA, } \\
\text { Nunes MLA, } \\
\text { kluppel BLP, } \\
\text { Medeiros SM, Sá } \\
\quad \text { LD }\end{array}$ & 2016 & Português & Scielo & $\begin{array}{l}\text { De acordo com a teoria de Leiniger com a formulação intercultural dos } \\
\text { direitos humanos, o cuidado é um ato que envolve valores, crenças, } \\
\text { conceitos e que deve ser dado sua respectiva importância. O } \\
\text { desconhecimento por parte do profissional de adquirir a cultura étnica, } \\
\text { enfraquece o cuidado em saúde e consequentemente a integralidade deste, } \\
\text { o distanciamento dos enfermeiros frente aos meios integrantes de saúde } \\
\text { geram dissimetrias no diálogo como profissional e cliente, não havendo o } \\
\text { olhar para cada usuário, como observar o ser individual e suas } \\
\text { particularidades. }\end{array}$ \\
\hline 8 & $\begin{array}{c}\text { Práticas } \\
\text { populares em } \\
\text { saúde indígena e } \\
\text { integração entre o } \\
\text { saber científico e } \\
\text { popular: revisão } \\
\text { integrativa }\end{array}$ & $\begin{array}{c}\text { Costa FAS, } \\
\text { Catanio PAG, } \\
\text { Aragão AEA, } \\
\text { Ponte HMS, } \\
\text { Fardin FP }\end{array}$ & 2016 & Português & BVS & $\begin{array}{l}\text { As práticas populares em saúde existem desde antigamente e continuam } \\
\text { até os dias de hoje. Mesmo com o avanço da medicina, a questão do } \\
\text { curandeirismo e os rituais religiosos continuam se destacando e } \\
\text { apresentando recursos através das suas crenças e culturas para a própria } \\
\text { população indígena, visto que entendem que os serviços de saúde } \\
\text { tradicional não os ofertam. A preservação das práticas e dos saberes } \\
\text { populares estipulado por um determinado grupo étnico possibilita o } \\
\text { aperfeiçoamento da equipe de saúde indígena na prevenção e promoção da } \\
\text { saúde no contexto da AP, com a contribuição até mesmo dos AIS } \\
\text { diversificando a atenção, a partir da implantação dos DSEI's. }\end{array}$ \\
\hline 9 & $\begin{array}{l}\text { Intermedicalidade } \\
\text { e protagonismo: a } \\
\text { atuação dos } \\
\text { agentes indígenas } \\
\text { de saúde } \\
\text { Munduruku da } \\
\text { Terra Indígena } \\
\text { Kwatá-Laranjal, } \\
\text { Amazonas, } \\
\text { Brasil. } \\
\end{array}$ & $\begin{array}{c}\text { Scopel, Daniel; } \\
\text { Paiva Dias- } \\
\text { Scopel Raquel; } \\
\text { Langdon Esther } \\
\text { Jean }\end{array}$ & 2015 & Português & Medline & $\begin{array}{l}\text { Os pajés das etnias indígenas possuem habilidades espirituais em busca da } \\
\text { cura, utilizando o saber cultural por meio de banhos de ervas, defumações, } \\
\text { massagens, rituais, ou seja, um conjunto de práticas que no ponto de vista } \\
\text { biomédico dificilmente seriam utilizados. O AIS tem uma participação } \\
\text { fundamental para aplicabilidade da medicina tradicional indígena e } \\
\text { saberes ofertados pelos médicos e enfermeiros, a atuação destes é } \\
\text { essencial devido ao acúmulo de saberes formado pela experiência na } \\
\text { função acrescentada na assistência realizada ao povo indígena, uma vez } \\
\text { que há precariedade nos serviços de saúde e acaba se tornando um agente } \\
\text { relevante as políticas de saúde indígena, pelas diversas atividades étnico- } \\
\text { culturais e cientificas vivenciadas. }\end{array}$ \\
\hline 10 & $\begin{array}{l}\text { Desafios na } \\
\text { formação de } \\
\text { enfermeiros } \\
\text { indígenas em } \\
\text { Mato Grosso, } \\
\quad \text { Brasil. }\end{array}$ & $\begin{array}{l}\text { Nascimento } \\
\text { VFD, Hattori } \\
\text { TY, Terças- } \\
\text { Trettel ACP. }\end{array}$ & 2020 & Inglês & PubMed & $\begin{array}{l}\text { A cultura indígena deve ser respeitada pelos profissionais de saúde e } \\
\text { trabalhada em conjunto com prescrições terapêuticas nas comunidades, } \\
\text { facilitando o processo de cura e valorização do sistema de saúde. O ensino } \\
\text { étnico indígena deveria ser implementado desde a formação de } \\
\text { profissionais, sejam eles médicos ou enfermeiros, pois as existências de } \\
\text { cotas para indígenas nas universidades garantem o acesso a graduação, } \\
\text { porém o reconhecimento da cultura é falho e fragilizado pela ciência, } \\
\text { desestimulando o indígena a desenvolver conhecimentos protegendo seus } \\
\text { valores e até mesmo contribuir com sua comunidade de origem. }\end{array}$ \\
\hline 11 & $\begin{array}{l}\text { Saúde e } \\
\text { problemas de } \\
\text { saúde na } \\
\text { perspectiva da } \\
\text { população } \\
\text { indígena da } \\
\text { Amazônia } \\
\text { peruana: um } \\
\text { estudo } \\
\text { qualitativo. }\end{array}$ & $\begin{array}{c}\text { Badanta B, } \\
\text { Lucchetti G, } \\
\text { Barrientos-Trigo } \\
\text { S, Fernández- } \\
\text { García E, } \\
\text { Tarriño- } \\
\text { Concejero L, } \\
\text { Vega-Escaño J, } \\
\text { de Diego- } \\
\text { Cordero R. }\end{array}$ & 2020 & Inglês & PubMed & $\begin{array}{l}\text { A mortalidade indígena ainda predomina nos índices por disparidades } \\
\text { culturais e falhas da atenção ofertada, por falta de equipes } \\
\text { multidisciplinares para a integralidade do cuidado e difícil acesso de } \\
\text { comunidades distantes. O principal acometimento de saúde em } \\
\text { comunidades indígenas provém das queimadas, atividades agrícolas e da } \\
\text { má ingesta de água pelos povos, causando assim diarreias, parasitoses, } \\
\text { doenças respiratórias, risco de picada por animais peçonhentos, entre } \\
\text { outros agravos. Mesmo que a medicina tradicional seja atualmente comum } \\
\text { entre a população indígena, a crença indígena ainda é predominante, pois, } \\
\text { os considerados curandeiros, sejam eles pajés ou algum outro titulado, } \\
\text { ainda hoje usam de terapias baseadas em plantas, rituais e técnicas para } \\
\text { prevenir doenças e tratar seus pacientes. }\end{array}$ \\
\hline 12 & $\begin{array}{l}\text { Diálogo e } \\
\text { respeito: bases } \\
\text { para a construção } \\
\text { de um sistema } \\
\text { intercultural de } \\
\text { saúde para as } \\
\end{array}$ & $\begin{array}{c}\text { Patiño Suaza Ae, } \\
\text { Sandín Vásquez } \\
\text { M. }\end{array}$ & 2014 & Espanhol & PubMed & $\begin{array}{l}\text { Segundo Patiño, a relação entre as equipes de saúde e os povos indígenas } \\
\text { não é tão harmoniosa devido à falta de resolubilidade da medicina } \\
\text { ocidental, por falta de rapidez no atendimento e medicações repetitivas, } \\
\text { fazendo com que isso gere uma desconfiança no tratamento e perda de } \\
\text { credibilidade da comunidade aos serviços de saúde, se tornando a última } \\
\text { alternativa a ser buscada pela saúde destes. A prática do planejamento }\end{array}$ \\
\hline
\end{tabular}




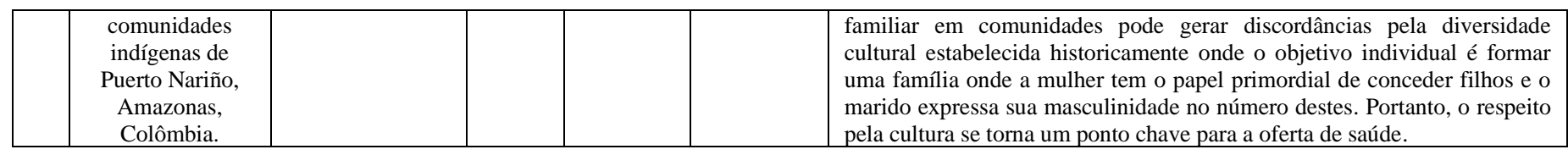

Fonte: Autores (2021).

No que concerne as atribuições do profissional de enfermagem, é primordial ter conhecimento quanto ao processo saúde-doença, pois, para o MS (2001) é considerado que a assistência prestada no meio das tribos indígenas tem impacto grandioso perante a qualidade de saúde e vida destas comunidades, assim como corrobora a ideia de capacitar um profissional que sustente um perfil que atenda as demandas e necessidades da comunidade onde atuam (Saviani, 2000), observando a realidade exposta cultural e étnica da sociedade nativa e transformando a formação transcultural dos enfermeiros. (Silva 2014) e (Lima 2005), concordam ao afirmar que a capacitação do profissional deve estar pautada a partir do modelo assistencial dentro da atuação da atenção básica, onde busca alcançar uma competência profissional para compreender as dimensões que compõem seu exercício de forma conjunta ou individual, capaz de conhecer e interpretar o processo de adoecimento e tratamento nos ambientes onde atuam.

Diante das políticas de saúde indígena descritas pelo Decreto n. ${ }^{\circ}$ 3.156, de 27 de agosto de 1999, que dispõe sobre as condições de assistência à saúde dos povos indígenas e decreta no Art. 1 1: "a atuação à saúde indígena é dever da União e será prestada de acordo com a Constituição e com a Lei no 8.080, de 19 de setembro de 1990, objetivando a universalidade, a integralidade e a equanimidade dos serviços de saúde". Mesmo que seja recomendado a toda à população indígena a atenção básica a saúde há as suas singularidades frente ao atendimento, ligados por exemplo as barreiras geográficas ou acesso; entretanto, observam-se as dificuldades no acesso para promover a equidade, integralidade no sistema de saúde indígena, observando também a falta de profissionais capacitados, alta rotatividade destes, assim como despreparo frente ao cuidado individual e familiar do cliente (Malouin et al., 2009).

A promoção a saúde ganhou força nos anos de 1990 no Brasil, quando houve uma influência no pensamento sanitário (Brasil, 2001). Mas, ainda nos dias atuais, a saúde requer um conjunto de atividades para promover saúde, capaz de melhorar as condições de bem-estar e acessos favoráveis a saúde (Czeresnia \& Freitas, 2009). Estudos apontam um cenário encontrado que emerge a importância de um olhar humanizado, constituídos com exemplos de valores à equidade, a seguridade social, para ter um comprometimento no sistema de saúde indígena, visto que o que encontramos diante do contexto atual é a pouca resolutividade de exercícios voltados para a saúde, assim como ações que aproximem a comunidade e a unidade básica de saúde, além de estratégias para informar a sociedade no que disser respeito ao acesso às informações (Oliveira et al., 2012).

Mesmo com a garantia de acesso integral a saúde, segundo o PNASPI, onde integra a diversidade social, histórica, política, cultural e o reconhecimento do povo, ainda há empecilhos frente ao acesso de saúde (Brasil, 2002, (Cardoso 2015), (Coimbra, 2001), (Tavares, 2010). Conforme Brasil (2007), as barreiras de acesso podem ser identificadas nos segmentos sociais organizacional, que são aqueles com características que impedem os esforços dos indivíduos em receber os cuidados como, por exemplo, a questão do horário de funcionamento de atendimento e o meio de geográfico, apresentados por exemplo na distância entre residência e centro de saúde e a falta de transportes ofertados para irem ao local de atendimento.

Diante do cenário encontrado com a falta de preparo por meio dos profissionais de saúde no Brasil, o SUS vem buscando conceder uma "cobertura" na saúde indígena, de forma universal, igualitária e atendendo a diversidade que deve-se ter ao assistir esta população, levando em consideração a apresentação da cultura e a língua nativa, conforme atribuições apresentadas por meio da Política Nacional de Assistência à Saúde dos Povos Indígenas (PNASPI) que busca proporcionar ações que evitem agravos a saúde desta sociedade (Brasil, 2007).

De acordo com o MS (2011), as práticas de cuidados devem considerar a diversidade, compreendendo seus diferentes 
significados e sentidos. De acordo com a Política Nacional de Atenção Básica à Saúde, devem ser consideradas ao prestar o cuidado, as condições culturais, econômicas, políticas, para tornar o atendimento à saúde mais eficaz. Os profissionais devem entender a competência técnica, assim como a valorização de culturas, suas crenças e seus costumes, para assim obter a possibilidade de exercer um melhor exercício de cuidado, tornando capaz de exercer uma função mais adequada, possibilitando que não haja enfraquecimento do cuidado em saúde, nem a integralidade do cuidado, nem o distanciamento entre equipe e usuário, (Lima, et al., 2016).

Dito por Oliveira e Leite (2011) e Torres e Silva (2011) desde as antigas civilizações, as práticas populares em saúde fazem parte da história da humanidade. Entretanto, os conhecimentos voltados a saúde indígena não se baseiam em mero achismo, mas em tradições de curas e rituais religiosos. Portanto, evidenciam a necessidade de obter-se conhecimento e conjunção entre o conhecimento ocidental e o conhecimento indígena, buscando a preservação e valorização da cultura e o aperfeiçoamento da equipe de saúde indígena.

$\mathrm{O}$ aspecto religioso era a proposta inicial para a educação nas comunidades indígenas anteriormente. Com o passar dos anos, houve um objetivo de incorporar no cotidiano dessas tribos a educação que os aproximava ao costume não indígena (Bergamaschi, et al., 2018). Quando estes começaram a adentrar nos espaços acadêmicos, encontraram-se dificuldades das universidades em dialogar com esses respectivos povos (Urquiza, 2014). Atualmente, nos modelos curriculares das maiorias das Instituições de Ensino Superior (IES), minimiza a possibilidade do abrangente ensino tradicional e possibilita uma construção em adquirir conhecimentos voltados para esses povos, trazendo uma perspectiva de assistências futuras diferenciadas nos serviços de saúde, buscando tornar-se capaz de criar um vínculo e respeito para as culturas, ensino étnico-indígena, assim como tentar fazer com que os indígenas que adentram em ensino superior sejam capazes de contribuir com a sua comunidade de origem (Urquiza, 2014, Hoefel, et al., 2015).

De acordo com pesquisas, muitas comunidades indígenas sofrem com as desigualdades sociais, tornando-se um fator para a predominância de taxas de mortalidade e menor acesso aos serviços de saúde (Vargas, et al., 2016); (Anderson, et al., 2016); (Diaz, et al., 2015); (Terán et al., 2016). Disposto por (Jauregui et al., 2011), os fatores culturais têm grande influência e nos mostram esses resultados de saúde através de estudos apresentados por meio das adversidades de saúde como problemas gastrintestinais, doenças infecciosas, entre outros (Bradford, et al., 2016; Lafontaine, 2018). Embora a assistência tradicional tenha se tornado parte do cotidiano da população indígena, os curandeiros tendem a usar suas técnicas e suas crendices por meio de curas espirituais, terapias por meio de plantas medicinais, entre outros costumes (Brierley,et al., 2014).

O direito a saúde dos povos indígenas relacionados a equipe-cliente, assim como a quantidade e qualidade de capacitação profissional, disponibilidade de acesso à tecnologia e modelo de saúde aplicados pelos profissionais de saúde explicam o porquê da divergência entre as equipes e a população indígena não ser harmoniosa, como por exemplo, acessar o serviço tardiamente (Torres, 2001). Segundo (Campos, 2003), a conscientização e aprendizagem por parte do profissional de enfermagem é crucial para compreender a visão dos indígenas, visto que seus ensinamentos norteiam a vida, a saúde, a morte, as doenças, as curas, as crendices.

O modelo diversificado de atenção com a implementação dos DSEI's teve grande auxílio com a contribuição dos AIS. No processo dos serviços prestados a saúde, incluem estratégias de capacitação de agentes comunitários. Os AIS têm uma conexão tanto da cultura indígena quanto das dos profissionais da atenção básica, portanto, entende-se que o cargo de agente de saúde traz consigo a ideia de ter um olhar interno a comunidade, sobre os problemas de saúde como das intervenções socioculturais e o processo saúde-doença (Dias, 2005; (Erthal, 2003); (Langdon, 2014); (Diehl, 2012); (Dias, 2015); (Pontes, AL et al., 2012) (Stauffer, \& Garnelo, 2012) 


\section{Conclusão}

No desencadeamento do trabalho da enfermagem, encontram-se diversos desafios quanto ao contexto de prestação na assistência indígena. Todavia, nos faz ressaltar a necessidade de uma averiguação no modelo da assistência ofertada as populações indígenas, havendo também capacitações à toda a equipe para um cuidado direcionado de forma direta ao cliente, fazendo com que se torne capaz de a equipe passar a ter uma visão não só geral, mas como um todo, onde seja possível reconhecer e entender as diversidades expostas no momento do atendimento ao usuário indígena.

Para uma melhor compreensão quanto a assistência ao indígena, recomenda-se pesquisas voltadas ao usuário da comunidade, bem como pesquisas direcionadas ao profissional que atua no contexto indígena, além de pesquisas no âmbito da atenção básica de forma geral, afim de adquirir conhecimento e informações sobre a temática e atentar-se a busca de uma melhor assistência voltada a este público para obter por partes dos governantes uma visão mais ampla sobre a realidade local dos profissionais de enfermagem.

Conclui-se uma importância quanto a melhoria nas implementações das políticas de forma compreensiva pelos gestores das três esferas (federal, estadual e municipal), principalmente as municipais ao implementar estas políticas no decorrer da assistência a comunidade local, ocasionando assim uma melhor qualidade na prestação de serviços ao cuidado à saúde indígena.

\section{Referências}

Anderson, I., Robson, B., Connolly, M., Al-Yaman, F., Bjertness, E., King, A., \& Yap, L. (2016). Indigenous and tribal peoples' health (The Lancet-Lowitja Institute Global Collaboration): a population study. The Lancet, 388(10040), 131-157.

Badanta, B., Lucchetti, G., Barrientos-Trigo, S., Fernández-García, E., Tarriño-Concejero, L., Vega-Escaño, J., \& de Diego-Cordero, R. (2020). Healthcare and Health Problems from the Perspective of Indigenous Population of the Peruvian Amazon: A Qualitative Study. International journal of environmental research and public health, 17(21), 7728

Bergamaschi, M., Doebber, M., \& Brito, P. (2018). Estudantes indígenas em universidades brasileiras: um estudo das políticas de acesso e permanência. Revista Brasileira de Estudos Pedagógicos, 99(251).

Bradford, L. E., Bharadwaj, L. A., Okpalauwaekwe, U., \& Waldner, C. L. (2016). Drinking water quality in Indigenous communities in Canada and health outcomes: a scoping review. International journal of circumpolar health, 75(1), 32336.

Brasil, Lei 7.498, de 25 de junho de 1986. Dispõe sobre a Regulamentação do Exercício da Enfermagem e dá outras providências. Brasília: Ministério da Saúde; 1986. http://www.planalto.gov.br/ccivil_03/leis/L7498.htm

Brasil. Ministério da Saúde; Fundação Nacional de Saúde. Política Nacional de Atenção à Saúde dos Povos Indígenas [Internet]. Brasília; 2002 http://bvsms.saúde.gov.br/bvs/publicações/política_saúde_indígena. Pdf

Brasil. Constituição (1988). Constituição da República Federativa do Brasil. Brasília, DF; Senado Federal:CentroGráfico, 1988. <http://www.planalto.gov.br/ccivel_03/constituição/constituição.htm>

Brasil. Ministério da Saúde; Fundação Nacional de Saúde. Política Nacional de Atenção à Saúde dos Povos Indígenas. (2a ed.), FUNASA; 2002.

Brasil. Ministério da Saúde (BR). Saúde indígena: etnodesenvolvimento das sociedades indígenas.); 2001.52 p.

Brasil. Ministério da Saúde; Conselho Nacional de Secretários de Saúde. Atenção Primária e Promoção da Saúde. CONASS; 2007.

Brasil. Portaria n. 2.656, de 17 de outubro de 2007. Dispõe sobre as responsabilidades na prestação da atenção à saúde dos povos indígenas, no Ministério da Saúde e regulamentação dos Incentivos de Atenção Básica e Especializada aos Povos Indígenas.

Brierley, C. K.; Suarez, N.; Arora, G.; \& Graham, D. (2014) Acesso à saúde e crenças sobre saúde dos povos indígenas na remota Amazônia Peru. Sou. J. Trop. Med. Hyg. 90, 180-183.

Borghi, A. C., Alvarez, A. M., Marcon, S. S., \& Carreira, L. (2015). Singularidades culturais: o acesso do idoso indígena aos serviços públicos de saúde. Revista da Escola de Enfermagem da USP, 49, 0589-0595.

Campos-Navarro R. Para um diálogo intercultural em saúde. Yolpahtli; 2003

Cardoso, M. D. (2015). Políticas de saúde indígena no Brasil: do modelo assistencial à representação política. Langdon EJ, Cardoso MD, organizadores, Saúde indígena: políticas comparadas na América Latina. Florianópolis: Ed. da UFSC, 83-106.

Ceccim, R. B., \& Merhy, E. E. (2009). Intense micropolitical and pedagogical action: humanization between ties and perspectives. Interface-Comunicação, Saúde, Educação, 13, 531-542.

Costa, F. A. S., Catanio, P. A. G., de Araújo Aragão, A. E., da Ponte, H. M. S., Fardin, F. P., \& de Araújo, L. M. (2016). Práticas populares em saúde indígena e 
integração entre o saber científico e popular: revisão integrativa. SANARE-Revista de Políticas Públicas, 15(2).

Coimbra Jr, C. E., \& Santos, R. V. (2001). Perfil epidemiológico da população indígena no Brasil: considerações gerais. Porto Velho: Centro de Estudos em Saúde do Índio de Rondônia, Universidade Federal de Rondônia/Rio de Janeiro: Escola Nacional de Saúde Pública, Fundação Oswaldo Cruz.

Conselho Federal De Enfermagem. Código de ética dos profissionais de enfermagem. COFEN; 2008.

Czeresnia, D., \& De Freitas, C. M. (2009). Promoção da saúde: conceitos, reflexões, tendências. SciELO-Editora FIOCRUZ.

Díaz, A., Arana, A., Vargas-Machuca, R., \& Antiporta, D. (2015). Situación de salud y nutrición de niños indígenas y niños no indígenas de la Amazonia peruana. Revista Panamericana de Salud Pública, 38, 49-56.

Dias-Scopel, R. P. (2005). O agente indígena de saúde Xokleng: por um mediador entre a comunidade indígena e o Serviço de Atenção Diferenciada à Saúdeuma abordagem da antropologia da saúde [Dissertação de Mestrado]. Florianópolis: Programa de Pós-Graduação em Antropologia Social, Universidade Federal de Santa Catarina.

Diehl, E. E., Langdon, E. J., \& Dias-Scopel, R. P. (2012). Contribuição dos agentes indígenas de saúde na atenção diferenciada à saúde dos povos indígenas brasileiros. Cadernos de Saúde Pública, 28, 819-831.

Erthal, R. M. C. (2003). A formação do agente de saúde indígena Tikúna no Alto Solimões: uma avaliação crítica. Coimbra Jr. CEA, Santos RV, Escobar AL, organizadores. Epidemiologia e saúde dos povos indígenas no Brasil. Rio de Janeiro: Editora Fiocruz, 197-215.

Ferreira, S. R. S., Périco, L. A. D., \& Dias, V. R. F. G. (2018). The complexity of the work of nurses in Primary Health Care. Revista brasileira de enfermagem, 71, 704-709.

Ferreira, L. O. (2013). The emergence of traditional indigenous medicine in the public policy field. História, Ciências, Saúde-Manguinhos, $20(1), 203-219$.

Fundação Nacional De Saúde. $4^{\text {a }}$ Conferência Nacional de Saúde indígena, Rio Quente-GO, relatório final. Brasília: Funasa, 2007.

FUNASA; Política Nacional de atenção à Saúde dos povos indígenas. 2002 <http://bvsms.saúde.gov.br/bvs/publicaços/política_saúde_indígena.pdf> .

Frontelmo, C. S. (2016). O papel do enfermeiro na assistência à população indígena no âmbito da atenção primária em saúde.

Garnelo, L., \& Pontes, A. L. (2012). Saúde Indígena: uma introdução ao tema. In Saúde Indígena: uma introdução ao tema (pp. 296-296).

Gelinski, C. R. O. G. (2011). A questão da co-responsabilidade prevista na estratégia saúde da família. Política \& Sociedade, 10(19), 97-

Giovanella, L., \& Mendonça, M. H. M. Atenção Primária à Saúde. In: Giovanella L, Escorel S, Lobato LVC, Noronha JC, Carvalho AI, organizadores. Políticas e Sistema de Saúde no Brasil. Rio de Janeiro: Editora Fiocruz; 2014. p.493-545. Livro

Greenwood, N., Habibi, R., Smith, R., \& Manthorpe, J. (2015). Barriers to access and minority ethnic carers' satisfaction with social care services in the community: a systematic review of qualitative and quantitative literature. Health \& social care in the community, 23(1), 64-78.

Hoefel, M. D. G. L., Severo, D. O., Díaz Bermúdez, X. P., Hamann, E. M., \& Carvalho, H. S. (2015). PET-Saúde Indígena UnB: construindo redes interculturais em saúde.

Jauregui, X.; Clavo, Z. M.; Jovel, E. M.; \&Pardo-de-Santayana, M. (2011), "Plantas con madre": Plantas que ensinam e orientam o processo de iniciação xamânica na Amazônia Centro-Leste peruana. J. Ethnopharmacol. 134, 739-752

Lafontaine, A. Disparidades de saúde indígenas: um desafio e uma oportunidade. Pode. J. Surg. 61, 300-301

Langdon, E. J. (2007). Diversidade cultural e os desafios da política brasileira de saúde do índio.

Langdon, E. J., Diehl, E. E., \& Dias-Scopel, R. P. (2014). O papel e a formação dos agentes indígenas de saúde na atenção diferenciada à saúde dos povos indígenas brasileiros. Teixeira CC, Garnelo L, organizadores. Saúde indígena em perspectiva: explorando suas matrizes históricas e ideológicas. Rio de Janeiro: Editora Fiocruz, 213-39.

Lima, M. D. R. D. A., Nunes, M. L. D. A., Klüppel, B. L. P., Medeiros, S. M. D., \& Sá, L. D. D. (2016). Atuação de enfermeiros sobre práticas de cuidados afrodescendentes e indígenas. Revista Brasileira de Enfermagem, 69, 840-846.

Lima, V. V. (2005). Competência: distintas abordagens e implicações na formação de profissionais de saúde. Interface-Comunicação, Saúde, Educação, 9, 369379 .

Luciano, G. dos S. O índio brasileiro: o que você precisa saber sobre os povos indígenas no Brasil de hoje (2006). Ministério da Educação. <http://Unesdoc.Unesco.org/imagens/0015/001545/154565 por.pdf>.

Malouin, R. A., Starfield, B., \& Sepulveda, M. J. (2009). Evaluating the Tools Used. Managed care.

Marinelli, N. P., Nascimento, D. F., Costa, A. I. P., Posso, M. B. S., \& Araújo, L. P. (2012). Assistência à população indígena: dificuldades encontradas por enfermeiros. Revista Univap, 18(32), 52-65.

Martins, J. C. L., Martins, C. L., \& Oliveira, L. S. D. S. (2020). Atitudes, conhecimentos e habilidades para o trabalho do enfermeiro no Parque Indígena do Xingu. Revista Brasileira de Enfermagem, 73.

Ministério Da Saúde. Secretaria de Políticas de Saúde. Projeto promoção da saúde. Ministério da saúde; 2001.

Ministério Da Saúde (BR). Fundação Nacional de Saúde. Política Nacional de Atenção à Saúde dos Povos Indígenas. 2002. 40p 
Ministério Da Saúde (BR). Saúde indígena: etno desenvolvimento das sociedades indígenas. 2001.52 p

Ministério da Saúde. Portal da Saúde (BR). Projeto de profissionalização dos trabalhadores da área de enfermagem. http://portal.saude.gov.br/portal/sgtes/

Ministério Da Saúde (BR). Fundação Nacional de Saúde. Política Nacional de Atenção à Saúde dos Povos Indígenas. 2002 [http://bvsms.saúde..gov.br/bvs/publicaçoes/política_saúde_indígena.pdf.

Nascimento, V. F. D., Hattori, T. Y., \& Terças-Trettel, A. C. P. (2019). Desafios na formação de enfermeiros indígenas em Mato Grosso, Brasil. Ciência \& Saúde Coletiva, 25, 47-56.

Patiño Suaza, A. E., \& Sandín Vásquez, M. (2014). Diálogo y respeto: bases para la construcción de un sistema de salud intercultural para las comunidades indígenas de Puerto Nariño, Amazonas, Colombia. Salud colectiva, 10, 379-396.

Pontes, A. L., Stauffer, A., \& Garnelo, L. (2012). Profissionalização indígena no campo da saúde: desafios para a formação técnica de Agentes Indígenas de Saúde. Garnelo L, Pontes AL, organizadores. Saúde indígena: uma introdução ao tema. Brasília: Secretaria de Educação Continuada, Alfabetização, Diversidade e Inclusão, Ministério da Educação, 265-88.

Rocha, E. S. C., Toledo, N. D. N., Pina, R. M. P., Fausto, M. C. R., D’Viana, A. L., \& Lacerda, R. A. (2020). Primary Health Care attributes in the context of indigenous health. Revista Brasileira de Enfermagem, 73(5).

Rodrigues, D. (2014). Proteção e assistência à saúde dos povos indígenas isolados e de recente contato no Brasil. OTCA.

Oliveira, J. W. B., Aquino, J. M., \& Monteiro, E. M. L. M. (2012). Promoção da saúde na comunidade indígena Pankararu. Revista Brasileira de Enfermagem, 65, 437-444.

Oliveira, S. C., \& Leite, J. C. Saber e poder popular no cuidado à saúde. Saberes e Práticas: Experiências em Educação Popular e Saúde. 2011;1(1):19-22.

Torres, R. A. M., \& Silva, M. R. F. Inter-relações entre comunicação, cultura e educação popular. Saberes e Práticas: Experiências em Educação Popular e Saúde. (2011)

Saviani, D. (1997). A nova lei da educação: trajetória, limites e perspectivas. Autores Associados.

Silva, N. C. D., Gonçalves, M. J. F., \& Lopes Neto, D. (2003). Enfermagem em saúde indígena: aplicando as diretrizes curriculares. Revista Brasileira de Enfermagem, 56, 388-391.

Silva, C. D. D. (2014). De improvisos e cuidados: a saúde indígena e o campo da enfermagem. In Saúde indígena em perspectiva: explorando suas matrizes históricas e ideológicas (pp. 181-212).

Souza, M. T., et al. Revisão integrativa: o que é? Como fazer isto? Einstein 8(1):102-106.

Scopel, D., Dias-Scopel, R. P., \& Langdon, E. J. (2015). Intermedicalidade e protagonismo: a atuação dos agentes indígenas de saúde Munduruku da Terra Indígena Kwatá-Laranjal, Amazonas, Brasil. Cadernos de Saúde Pública, 31, 2559-2568.

Tavares, F. G. (2010). Epidemiologia da hipertensão arterial e níveis tensionais em adultos indígenas Suruí, Rondônia, Brasil (Doctoral dissertation).

Terán-Hernández, M., Díaz-Barriga, F., \& Cubillas-Tejeda, A. C. (2016). Diagnóstico de salud y percepción de riesgos, elementos clave para una propuesta de intervención en comunidades indígenas en México. Revista Panamericana de Salud Pública, 39, 106-114.

Torres C. Equidade em saúde vista de uma perspectiva étnica. Revista Pan-Americana de Saúde Pública. 10 (3): $188-201$

Urquiza, A. H. A. (2014). Direitos humanos e educação intercultural: as fronteiras da exclusão e as minorias sub-representadas-os indígenas no ensino superior. Série-Estudos-Periódico do Programa de Pós-Graduação em Educação da UCDB, 141-154.

Vargas-García, S.; Berúmen-Burciaga, LV; Arias-Pacheco, I.; Mejia-Mejia, Y.; Realivázquez-Pérez, L.; \& Portillo-Sánchez, R. Determinantes sociais do cuidado comunitário: percepções da enfermeira e indígena Raramuris. CULCyT Cult. Científica Tecnológica 57, 310-318.

Vieira, H. T. G., Oliveira, J. E. L., \& Neves, R. C. M. A Relação de intermedicalidade nos Índios Truká, em Cabrobó - Pernambuco. Saúde Soc. 22(2):566-74. 